\title{
Structure Design Fused with Electronic Devices of Holonic Manipulator*
}

\author{
Yasushi IKEI**, Michitaka HIROSE*** and Takemochi ISHII ${ }^{* * * *}$
}

\begin{abstract}
A holonic manipulator has many electronic devices such as control computers and sensors among its local mechanical components. This kind of manipulator design has three major advantages: reduction of wire harness between devices, high software productivity, and fault tolerability of the system. In this paper, we first represent two actually developed holonic manipulators. Then we compose a simple structural model of the manipulator focusing particularly on the constituent component mass so that the efficiency ratio of payload mass to total manipulator mass can be analyzed. This efficiency ratio exhibits a saturated increase consistent with the payload mass, which is due to DC servomotor mass/power characteristics and mechanical structure mass/ stress characteristics. For the improvement of the efficiency ratio, the fused structure design has been employed mainly between heat sinks or support members of the computer and mechanical structure. The achieved improvement is estimated with the model using a mass fusion function.
\end{abstract}

Key Words : Mechatronics, Robotics, Control Device, Manipulator, Fused Structure Design, Payload-Deadload Ratio

\section{Introduction}

The present robot manipulator system is com. posed of elements of two kinds. One is a mechanical element which forms robot mechanisms such as a structural member, a joint mechanism, or a power generation and transmission mechanism. The other is an electronic component such as a motor drive controller of a joint, a controller of a sensor attached to various parts, or a computer that generally manages these devices. The two kinds of elements are designed independently before they are connected to each other by wiring when the robot is finally assembled.

One of the problems with this conventional design is the connection wirings when we are to equip a robot with additional intelligence. In order for a robot to become more intelligent, it is necessary for it to

* Received 21st June, 1991. Paper No. 89-1333B

** Faculty of Engineering, Osaka University. 2-1 Yamadaoka Suita-shi, Osaka 565, Japan

*** Faculty of Engineering, The University of Tokyo. 73-1 Hongo Bunkyo-ku, Tokyo 113, Japan

**** Faculty of Environmental Information, Keio University. 5322 Endoh, Fujisawa 252, Japan process a large quantity of information about its environment as well as itself. It is also imperative for the robot to exercise more degrees of freedom in control than with which it is equipped at present, including more precise control of its fingers so as to accomplish more precise manipulation of objects. This requires that various sensors and motors be equipped in large numbers at each part of the robot, especially at the hand. Thus, the wirings needed to connect the parts with their controllers will become quite substantial. Since securing the reliability of many connectors is difficult to accomplish, maintainability certainly deteriorates in accordance with the increase in wire junctions. Naturally, reliability of the robot system decreases because most system failures occur at connections between parts. Moreover, it is required that robot control incorporates the rigidity and weight of wiring when the amount of wiring increases significantly. However, since the modeling of a wire harness is not necessarily easy to complete, precise motion trajectory of the robot cannot be achieved.

The authors are developing a holonic manipulator which has many small computers embedded in each part of the mechanical structure to solve the problems 
that arise in the realization of an intelligent $\operatorname{robot}^{(1),(2)}$. Most of the wirings that result from present structural design where a mechanical element and a electronic element are divided completely are notably reduced by being localized by the design developed here. The wiring traversing a movable part of a joint is limited to a multiplexed communication network and a power line which connect embedded computers. In this article, we first describe two design instances of the holonic manipulator which is equipped with computers at every joint part. By embedding electronic parts, the possibility arises that the mass (weight) ratio of maximum payload to total manipulator mass may decrease. We then estimate the ratio improvement expected by this design of fused parts based on the structural model of the manipulator.

Table 1 Specifications of manipulator No. 1

\begin{tabular}{|c|c|}
\hline $\begin{array}{l}\text { Hechanical configuration } \\
\text { Degrees of freedon }\end{array}$ & $\begin{array}{l}\text { Articulated-type anipulator } \\
6(R-P-R-P-R-P-)\end{array}$ \\
\hline $\begin{array}{l}\text { Degrees of Ireedon } \\
\text { Gripper wechanisa }\end{array}$ & Two-fingered hand ( 1 d.o.f $)$ \\
\hline Max. load & Approximately $0.5 \mathrm{~kg}$ \\
\hline $\begin{array}{l}\text { Joint driving echaniss } \\
\text { Controller }\end{array}$ & $\begin{array}{l}\text { DC servouotor + reduction gears } \\
\text { Joint embedded controller } \\
\cdot \text { i } 8751 \text { (8bit CPU, AKB EPROM) } \\
\text { - 10bit A/D converter }\end{array}$ \\
\hline Control odes & $\begin{array}{l}\text { Three odes of position, veloc- } \\
\text { ity and dynanic control }\end{array}$ \\
\hline Controller connection & Bus network \\
\hline Comanication speed & $125 \mathrm{Kbps}$ \\
\hline Comand transuission & Asynchronous wessage of packets \\
\hline
\end{tabular}

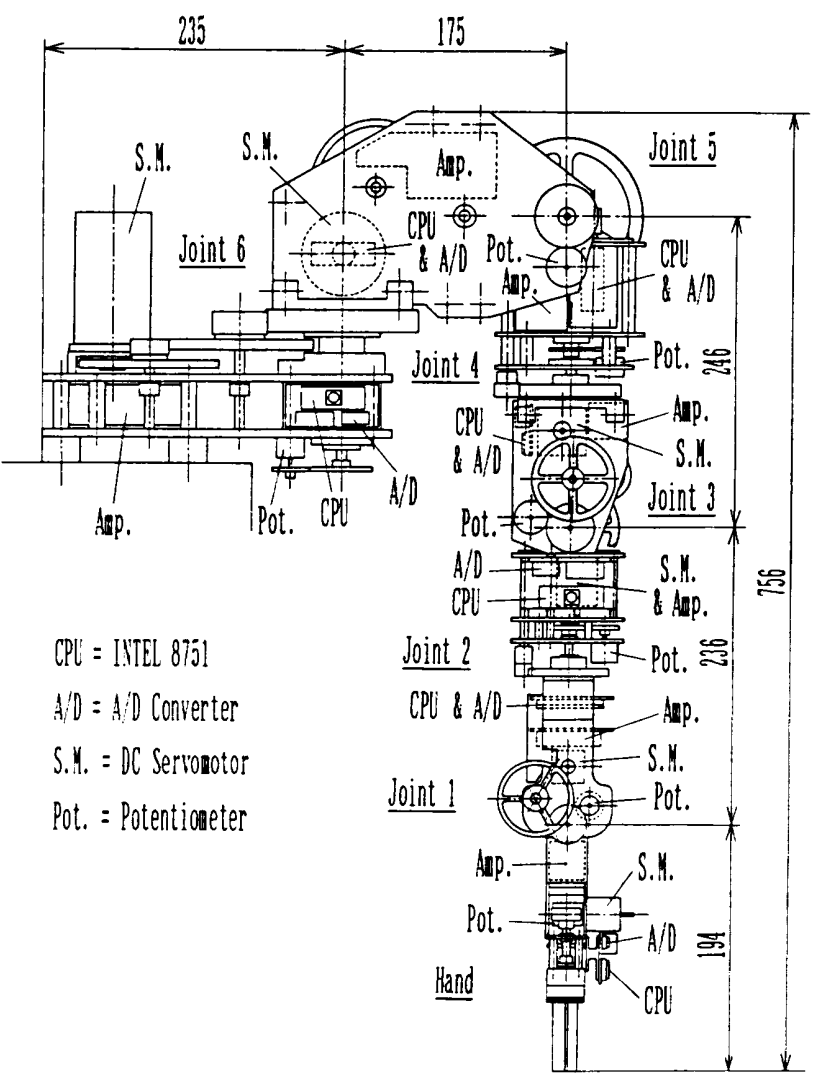

Fig. 1 Structure of manipulator No. 1

\section{Structural Design of Holonic Manipulator}

\subsection{Design of holonic manipulator No. 1}

Figure 1 shows the structure of holonic manipulator No. 1, and its specifications are listed in Table 1. Manipulator No. 1 has six-degree-of-freedom (d. o. f.) rotating joints and one d. o.f. hand. Each joint and the hand are equipped with its own embedded control computer. The computer not only executes the joint servo autonomously as a complete unit, but plays a slavish part of the total arm trajectory control according to the messages arriving through the communication network. This characteristic of the joint element is the source of its name 'holonic'.

Connection wirings between joints are reduced to seven at most, including power lines, by linking them to a communication network which has bus topology. The wires originating from each joint denoted in Fig. 2 are such connection wirings which comprise two motor power lines, two shared power lines for the control computer and sensors, and three communication lines.

A computer embedded in each part has the minimum composition for joint control, which includes a single-chip CPU, an A/D converter, and its power supply integrated in a $38 \times 68 \mathrm{~mm}^{2}$ board. A motor drive amplifier is also embedded in the joint to form a simple bridge circuit by discrete transistors, which switches the power output according to a PWM signal from the CPU.

Table 2 shows the constitutional mass of the manipulator. The maximum ratio of the embedded electronic part mass to the whole joint mass reaches $35 \%$ at the first joint. This implies that the ratio has already required such intensive consideration at the time of design that the electronic part mass cannot be ignored, although the embedded electronic parts are not very numerous in this design instance.

\section{2 Design of holonic manipulator No. 2}

The structure of manipulator No. 2 is shown in Figs. 3 and 4, and its specifications are listed in Table

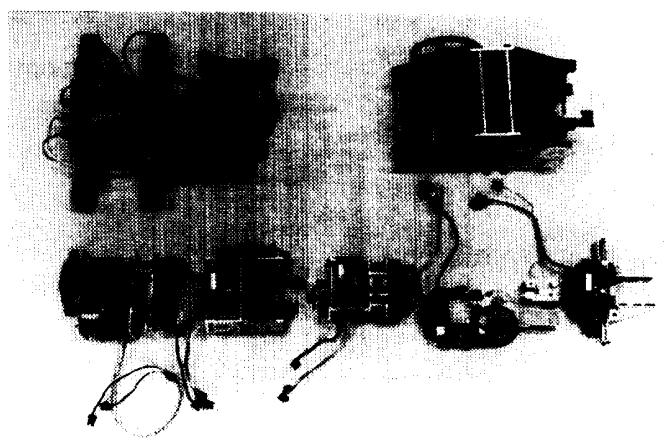

Fig. 2 Decomposed joints of manipulator No. 1 
3. The embedded computer of manipulator No. 2 is designed to have an extremely large computational capacity for real-time solution of manipulator dynamics. Since the mass and the volume of the computer must be augmented in accordance with the capacity increase, the computer parts and mechanical parts are designed from the beginning as a fused form

Table 2 Constitutional mass of manipulator No. 1

\begin{tabular}{|c|c|c|c|c|c|c|c|}
\hline & \multirow{2}{*}{$\begin{array}{c}\text { Total aass } \\
|\mathrm{g}|\end{array}$} & \multicolumn{2}{|c|}{ Mechanical parts $\mid \mathrm{g}$ ] } & \multicolumn{3}{|c|}{ Electronic parts [B ] } & \multirow{2}{*}{$\begin{array}{l}\text { Ratio of } \\
\text { electronic } \\
\text { parts }|\%|\end{array}$} \\
\hline & & structure & Motor & $\begin{array}{l}\text { C PU } \\
\text { board }\end{array}$ & $\begin{array}{l}\text { Potent1- } \\
\text { ometer }\end{array}$ & Aaplifier & \\
\hline Hand & 578 & 397 & 80 & 71 & 13 & 17 & 17.5 \\
\hline Joint 11 & 563 & 234 & 130 & 59 & 13 & 127 & 35.3 \\
\hline Joint 12 & 780 & 423 & 130 & 59 & 13 & 155 & 29.1 \\
\hline Joint 13 & 1370 & 753 & 420 & 65 & 13 & 119 & 14.1 \\
\hline Joint 14 & 1600 & 959 & 420 & 65 & 13 & 143 & 13.8 \\
\hline Joint is & 4155 & 2639 & 1100 & 64 & 13 & 340 & 10.0 \\
\hline Joint 16 & 7765 & 6289 & 1100 & 63 & 13 & 290 & 4. 7 \\
\hline Total & 16811 & 11704 & 3380 & 446 & 91 & 1191 & 10.3 \\
\hline
\end{tabular}

Table 3 Specifications of manipulator No. 2 to compensate for this augmentation. The embedded computer is composed of four hybrid ICs (made of $50 \times 75 \times 1 \mathrm{~mm}^{3}$ ceramic board) as shown in Fig. 5 : two CPU boards, a ROM board, and a shared memory board. They are put together, respectively, with a duralumin structural support member (Fig. 6) which also functions as a heat sink. They are then connected by two FPC (flexible printed circuit) boards at 50 lead wires attached on both sides after being piled up as shown in Fig. 7. A motor drive amplifier is also embedded in the structure after fabrication into a hybrid IC (made of $38 \times 55 \times 1 \mathrm{~mm}^{3}$ aluminum board). This bulk of the computer part occupies the primary ratio of manipulator volume and forms a contour line of the manipulator.

The embedded computers at the joints and hand, each of which has two CPUs, are connected to each other by a loop network. This network is actually composed of a serial concatenation of small loops, each of which contains four adjacent CPUs, since the

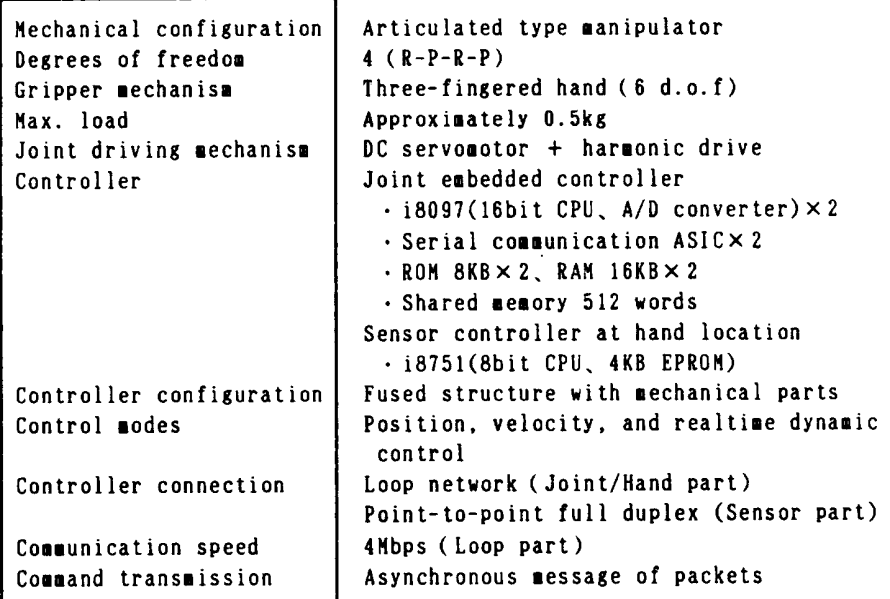

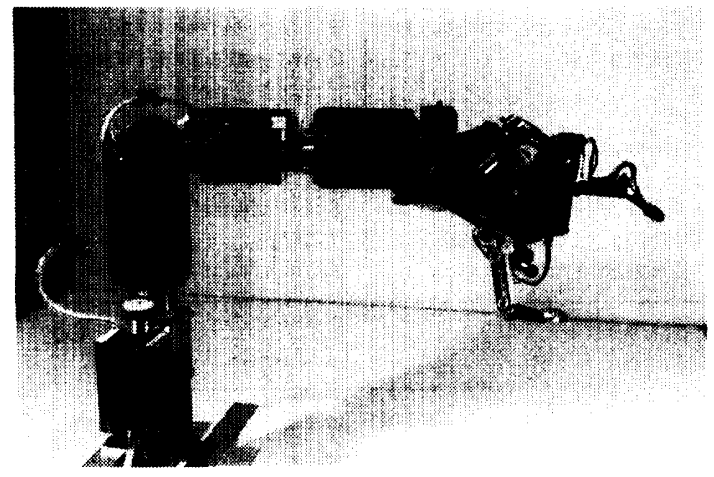

Fig. 3 Manipulator No. 2

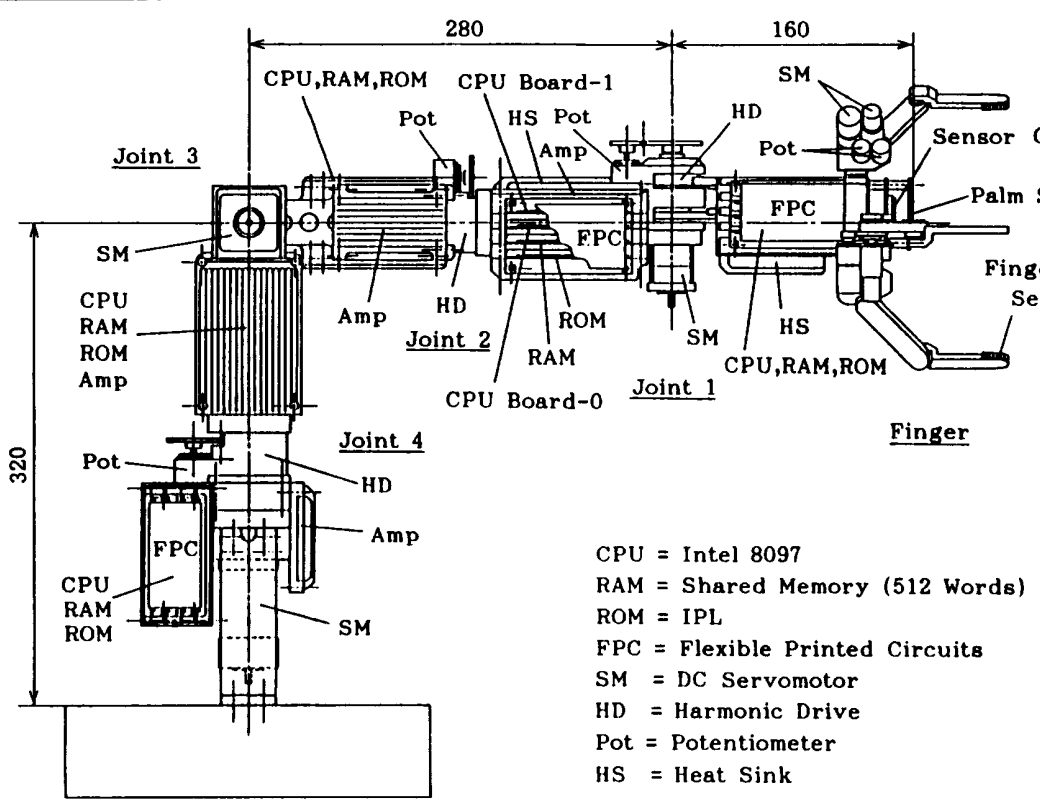

Fig. 4 Structure of manipulator No. 2 
two CPUs in one joint utilize a shared memory to communicate with each other. The communication among CPUs is executed by selecting the shortest path in the network. The number of connection wirings between the joints is ten at most : six for a round trip of the communication line and four for two systems of the power line as in manipulator No. 1. The effect of wire reduction is notable since more than 80 proximity sensors are equipped at the fingertips and palm, in addition to the motors and potentiometers for the six degrees of freedom of the three fingers of the hand.

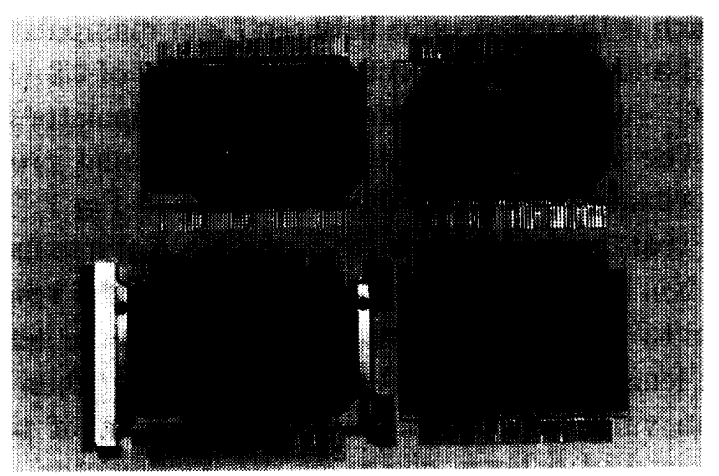

Fig. 5 Embedded computer of manipulator No. 2

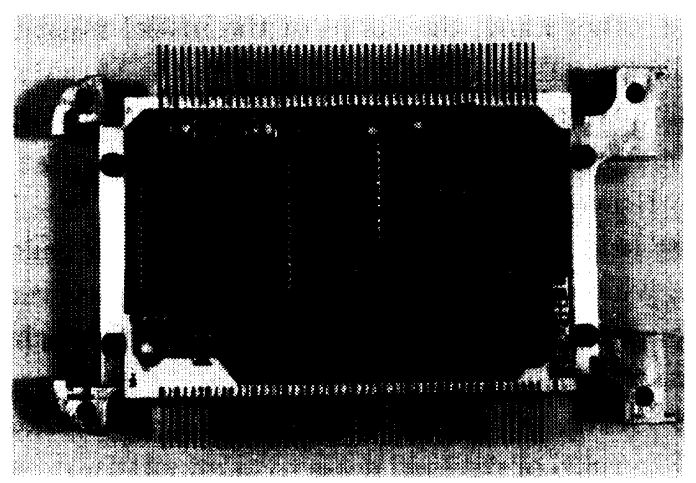

Fig. 6 CPU board

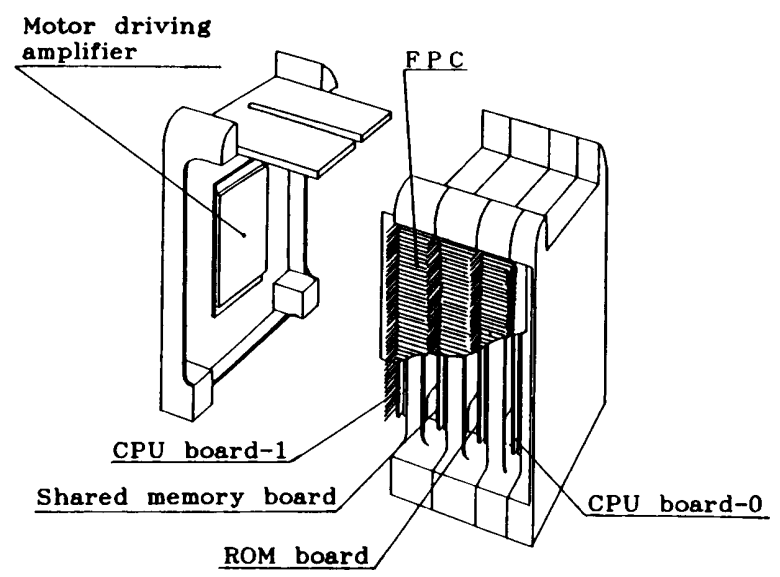

Fig. 7 Structure of an embedded computer and an amplifier (heat sink omitted)
Table 4 shows the constitutional mass of manipulator No. 2. Manipulator No. 2 has some parts that cannot be classified definitely, as in the case of manipulator No.1, owing to its fused structure design. These parts correspond to support members which also serve as a computer board or as a heat sink of an amplifier. They are included in the mechanical part mass in Table 4 . In the table, therefore, the ratio of pure electronic parts is given only for reference. Although the hand has a massive amount of additional mechanical parts in the fingers, the ratio of pure electronic parts already amounts to about $27 \%$ of the largest figure at the hand since it is furnished with many sensors.

\section{Design Evaluation by Structural Model}

\section{1 Structural model of holonic manipulator}

We describe the structure of the holonic manipulator mentioned in the previous section by a simplified model (Fig. 8). The nomenclature is as follows:

$M_{0}$ : payload mass including a hand $\mathrm{kg}$

$M_{i}:$ total mass of the $i$-th joint $\mathrm{kg}$

$M m_{i}:$ motor mass of the $i$-th joint $\mathrm{kg}$

$M s_{i}$ : structural member mass of the $i$-th joint $\mathrm{kg}$ $M e_{i}$ : electronic part mass of the $i$-th joint $\mathrm{kg}$

$M h_{i}$ : heat sink mass of a motor drive amplifier of the $i$-th joint $\mathrm{kg}$

$P_{i}:$ motor output power of the $i$-th joint W

$T_{i}$ : joint torque of the $i$-th joint $\mathrm{Nm}$

$V_{d}$ : payload velocity $\mathrm{m} / \mathrm{s}^{2}$

$\dot{V}_{\alpha}$ : payload acceleration $\mathrm{m} / \mathrm{s}^{2}$

$l$ : length of the link $\mathrm{m}$

The manipulator is modeled by joints with a centralized mass and links which have the same length and negligible mass. The centralized total mass

Table 4 Constitutional mass of manipulator No. 2

\begin{tabular}{|c|c|c|c|c|c|c|c|}
\hline & \multirow{2}{*}{$\begin{array}{l}\text { Total } \\
\text { wass } \\
{[\mathrm{g}]}\end{array}$} & \multicolumn{2}{|c|}{ Mechanical parts[s] } & \multicolumn{3}{|c|}{ Pure electronic parts[s] } & \multirow{2}{*}{$\begin{array}{l}\text { (cf.) Ratio } \\
\text { of } \\
\text { pure } \\
\text { electronic } \\
\text { parts } \mid \%]\end{array}$} \\
\hline & & Structure & Motor & $\begin{array}{l}\text { CPU } \\
\text { Board }\end{array}$ & $\begin{array}{l}\text { Potentio- } \\
\text { ueter etc. }\end{array}$ & $\begin{array}{l}\text { Aapli- } \\
\text { fier }\end{array}$ & \\
\hline Hand & 750 & 339 & 210 & 80 & $\begin{array}{c}\text { (Sensor) } 71 \\
30\end{array}$ & 20 & 26.8 \\
\hline Joint 1 & 745 & 508 & 130 & 80 & 13 & 14 & 14.4 \\
\hline Joint 12 & 780 & 548 & 130 & 80 & 13 & 14 & 13.8 \\
\hline Joint 13 & 1340 & 813 & 420 & 80 & 13 & 14 & 8.0 \\
\hline Joint 4 & 1680 & 1153 & 420 & 80 & 13 & 14 & 6.4 \\
\hline Total & 5295 & 3361 & 1310 & 400 & 153 & 76 & 11.9 \\
\hline
\end{tabular}

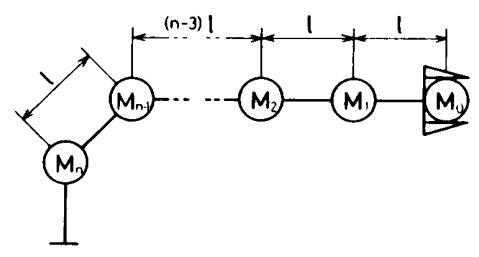

Fig. 8 Structural model of holonic manipulator 
$M_{i}$ of the joints is the sum of four kinds of masses of the constitutional part: $M_{i}=M m_{i}+M s_{i}+M e_{i}+M h_{i}$. Each mass of the constitutional part is estimated with the following assumptions.

(1) Motor mass $M m_{i}$ - The mass $M m_{i}$ of the $\mathrm{DC}$ servomotor is considered to have a nearly proportional relation to its power $P_{i}$ according to a survey of market products ${ }^{(3)}$. We use the next empirical formula derived from our survey to obtain the motor mass :

$$
M m_{i}=0.0501 \cdot P_{i}^{0.88} \text {. }
$$

(2) Motor power $P_{i}$ - Motor power is determined by the power to be applied to a payload. Where angular velocity and angular acceleration of the $i$-th joint are denoted as $\omega_{i}$ and $\dot{\omega}_{i}$, respectively, the required power to drive only $M_{0}$ is the following:

$$
\begin{aligned}
& P_{i 0}=M_{0} \cdot \dot{V}_{d} \cdot V_{d} \\
& \quad=M_{0} \cdot(i l)^{2} \cdot \dot{\omega}_{i} \cdot \omega_{i} .
\end{aligned}
$$

The power $P_{i}$ is given by the next formula, where the joint $i$ drives all the centralized masses located in one direction of the hand when all the joints are extended.

$$
\begin{aligned}
P_{i} & =\sum_{j=1}^{i} M_{i-j} \cdot(j l)^{2} \cdot \dot{\omega}_{i} \cdot \omega_{i} \\
& =\dot{V}_{d} V_{d} / i^{2} \cdot \sum_{j=1}^{i} M_{i-j} \cdot j^{2}
\end{aligned}
$$

(3) Joint torque - Joint torque is calculated by the next formula with motor power $P_{i}$ and angular velocity $\omega_{i}$ of the joint :

$$
T_{i}=P_{i} / \omega_{i}=P_{i} \cdot i \cdot l / V_{d}
$$

(4) Joint structural member and reduction gear mass - The mass of a joint structural member and a reduction gear of a motor are estimated together since they cannot be definitely discriminated from each other. In the strength design of a reduction gear shaft based on the maximum shearing stress estimation, the diameter $d$ of a shaft is calculated by the next formula, where $\tau$ denotes permissible shearing stress and $T e$ represents an equivalent torsional moment :

$$
d=\left(16 \cdot T e_{i} /(\pi \tau)\right)^{1 / 3} .
$$

If the structural member is evaluated from only shaft material, its mass is given as

$$
M s_{i} \sim d^{2} \cdot l \sim T e_{i}^{2 / 3} \cdot l .
$$

Moreover, if bending stress imposed on the member is evaluated, we also obtain the same result. The cogwheels which occupy a large proportion of the reduction gear mass have a proportional relationship between their mass and driving torque, according to the market $\operatorname{data}^{(3)}: M s_{i} \sim T e_{i}^{1.0}$. Since this gives an appropriate value so that the power of $T e_{i}$ selected as the mean value, we estimate structural mass by the next formula, where $r$ is a proportional constant :

$M s_{i}=r \cdot T e_{i}^{0.833} \cdot l$.

(5) Motor drive amplifier heat sink mass Calorific value to be radiated is proportional to the power consumption of a joint. Moreover, heat radia. tion capacity is also nearly proportional to the mass of a heat sink. Thus, the mass is obtained by the next formula, where $s$ is a proportional constant:

$$
M h_{i}=s \cdot P_{i} \text {. }
$$

Proportional constants of $r$ and $s$ are determined as $r=1.3$ and $s=0.022$ according to actual measurement on the prototypes. Each joint mass estimated based on these formulae is shown in Fig. 9 with real measured values of manipulators No. 1 and No. 2. The data used in the estimation are the following: payload of $1.0[\mathrm{~kg}]$, payload velocity of $0.5[\mathrm{~m} / \mathrm{s}]$, payload acceleration of $9.81\left[\mathrm{~m} / \mathrm{s}^{2}\right]$, link length $l$ of $0.135[\mathrm{~m}]$ (which is the mean value between $l \mathrm{~s}$ of manipulators No. 1 and No. 2), and electronic part mass of $0.8[\mathrm{~kg} /$ joint] (which is equivalent to that of manipulator No. 1). The joints of No. 1 through No. 4 exhibit proper accordance with actual measured data in Fig. 9. The fact that the actual joints No. 5 and No. 6 of manipulator No. 1 show larger values than the model results from two design considerations. One is that the actual link length of these portions is much longer than the mean value used in the model on account of total geometrical balance design of the manipulator, and the other is that design strength is overestimated toward the safety side so as not to reduce the mass. On the other hand, the curve of the model must indicate larger values if the model utilizes the actual data of manipulator No. 2 which has a longer link length of $0.15[\mathrm{~m}]$ and the bigger electronic part mass of 0.2 [kg]. However, the mass of manipulator No. 2 is almost held at the same value as that of manipulator No. 1, which is derived from the effect of mass reduction by the fused design described above. This point is discussed again in section 3. 3 .

\section{2 Ratio of payload mass to manipulator mass}

The ratio of payload mass to manipulator mass of the above model is shown in Fig. 10 which has the two parameters of payload mass and link length. The ratio

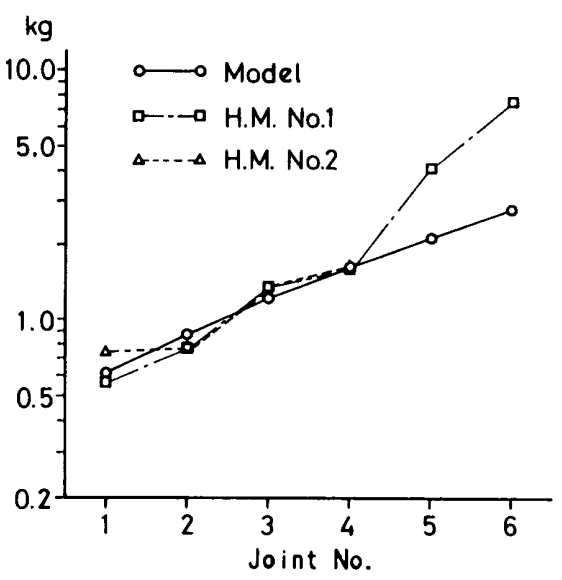

Fig. 9 Mass of the joints 
of payload mass to manipulator mass, which indicates its efficiency as a transfer machine, increases in accordance with augment of payload, and the increase rate has a tendency for saturation. This reflects a nonlinear relationship between the desired output and the mass increase rate of structural members with a motor. It is concluded from the figure that payload should be appropriately large if no severe limitation to the total mass increase is imposed, since sufficient efficiency cannot be achieved if the payload is too small. It is also found that the efficiency can be easily improved if the movable region of the manipulator allows for abatement because it is almost inversely proportional to the link length. The ratio has a value of 0.107 in the case of the model data shown above. In addition, the required payload velocity and acceleration are constant in this figure. If these values are changed in proportion to the link length, the curves will expand upwards and downwards from the referenced curve of the link length fixed in the center. However, in that case, the general tendency that the curves show is identical to that in this figure.

\section{3 Improvement of mass ratio by fused design}

The mass ratio related above can be improved by a fused design that merges structural members with electronic parts such as a computer and a heat sink of a motor drive amplifier. The quantity of the improvement can be estimated by applying the fused design of manipulator No. 2 to the model.

Here, we calculate the mass $M_{c}$ of the part which is designed from two parts of mass $M_{a}$ and $M_{b}\left(M_{a} \leqq\right.$ $M_{b}$ ) in a fused manner by the next formula :

$$
M_{c}=\left(M_{a}+M_{b}\right) \cdot F_{u}\left(M_{a} / M_{b}, \nu\right)
$$

where $F_{u}$ is a fusion function that removes the common part between $M_{a}$ and $M_{b}$ by the rate $\nu$. The function is defined as

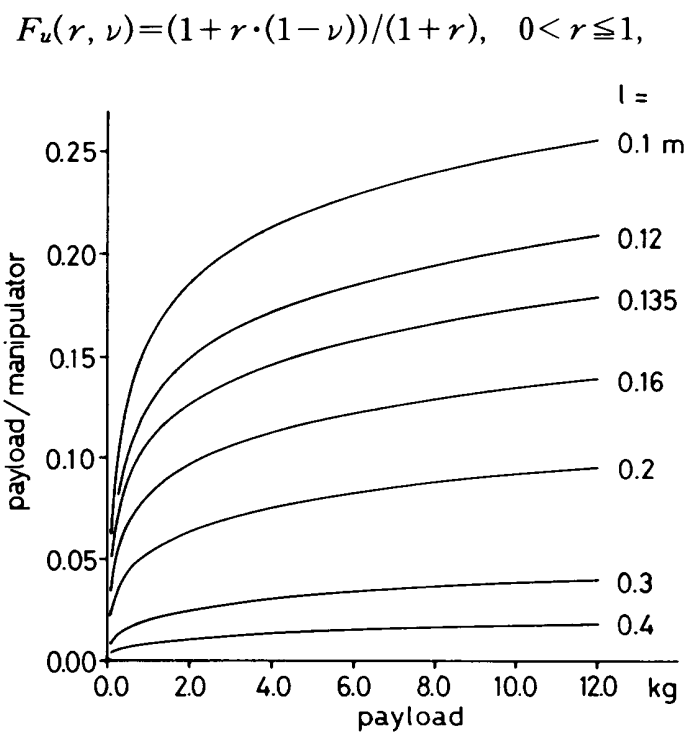

Fig. 10 Ratio of payload mass to manipulator mass where $\nu$ is a fusion rate which is defined as $0 \leqq \nu \leqq 1$. If $\nu=1$, then the maximum mass $M_{a}$ is reduced.

In the design of manipulator No. 2, support members of a computer which work also as a heat sink, and an amplifier heat sink are designed in this fused manner with the joint support members. The computer mass, that is, approximately $0.2[\mathrm{~kg}]$ for one joint, can provide half of itself as structural members of the link. The amplifier heat sink can provide almost all of its mass. When these masses are fused with $M s_{i}$, the total mass is calculated by the next formula:

$$
\begin{aligned}
M_{i} & =M m_{i}+M e_{i} \cdot 0.5+\left(M h_{i}+M e_{i} \cdot 0.5\right. \\
& \left.+M s_{i}\right) \cdot F_{u}\left(\left(M h_{i}+M e_{i} \cdot 0.5\right) / M s_{i}, \nu\right) .
\end{aligned}
$$

The ratio of payload mass to manipulator mass estimated by the formula is shown in Fig. 11, where payload mass and fusion rate $\nu$ are the parameters. The link length is 0.15 [m] and computer mass is $0.2[\mathrm{~kg}]$. The mass ratio is augmented by the fused design at the rate of from $25 \%$ to $40 \%$, and the increase rate is more significant for the lighter payload to promote efficiency improvement. In the case of manipulator No. 2, whose payload mass is $1.0[\mathrm{~kg}]$, the mass ratio is raised from $8.1 \%$ to $10.4 \%$, which corresponds to the manipulator mass reduction of $12 \%$ from $12.3[\mathrm{~kg}]$ to $9.6[\mathrm{~kg}]$.

Figure 12 shows the total mass of each joint. The mass difference between joints No. 1 and No. 2 of manipulator No. 2 is smaller than that of the model since the two joints are designed in almost the same configuration. However, it is observed that the manipulator mass is reduced to nearly the fusion rate of $\nu=$ 1.0 on an average.

\section{Conclusions}

In this paper, we demonstrated embodiment examples of a holonic manipulator which has a control computer within every joint and within the hand in order to equip a robot manipulator with higher

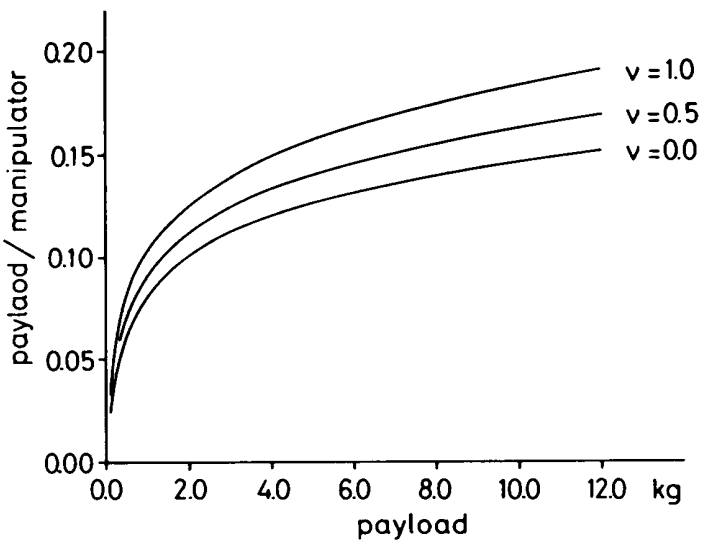

Fig. 11 Effect of mass ratio improvement by the fused design 


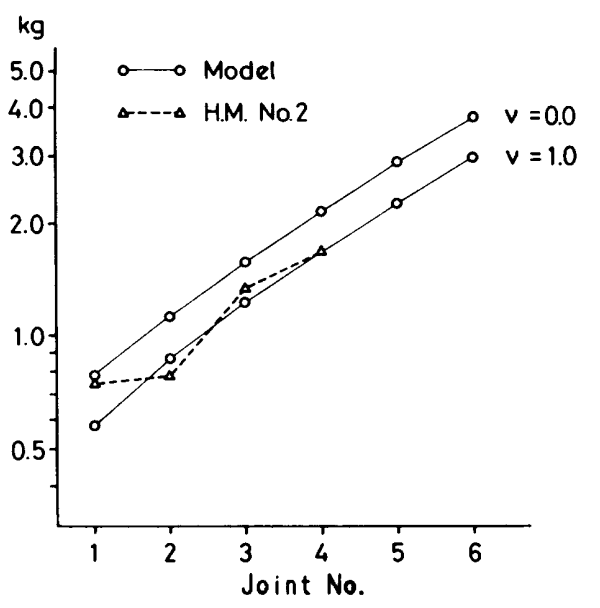

Fig. 12 Joint mass reduction by the fused design

intelligence. Moreover, we estimated the fused design of electronic parts focusing on the efficiency of payload-deadload mass ratio by a simplified structural model of the manipulator. Consequently, it is presumed that the efficiency has been improved by $2.3 \%$ and the total mass has been reduced by approximately $12 \%$.

\section{References}

(1) Hirose, M., Ikei, Y. and Ishii, T., Development of holonic manipulator, Proc. Japan-U.S. A. Symp. on Flexible Automation, (1986), p. 269.

(2) Hirose, M., Ishii, T., Ikei, Y. and Amari, H., Software environment for holonic manipulator, U.S. A.-Japan Symp. on Flexible Automation, (1988), p. 317.

(3) Takano, M., Design of robot mechanisms, J. of Robotic Society of Japan, (in Japanese), Vol. 4, No. 4 (1986), p. 394. 